\title{
Correlations and Fluctuations: Status and Perspectives
}

\author{
V. Koch \\ Nuclear Science Division \\ Lawrence Berkeley National Laboratory \\ Berkeley, CA 94720, USA \\ E-mail: vkoch@lbl.gov
}

\begin{abstract}
We will provide an overview of the physics which can be addressed by studying fluctuations and correlations in heavy ion collisions. Observables, which have been discussed in the literature will be briefly reviewed and put in context with experiment and information from Lattice QCD. Special attention will be given to the QCD critical point and the first order co-existence region.
\end{abstract}

\section{Introduction}

The knowledge of fluctuations and correlations is essential in characterizing a physical system. It will give information about the effective degrees of freedom. In addition, the susceptibilities, which characterize the correlations and fluctuations, also determine the response of the system to small external forces.

A prominent example where the measurement of correlations has lead to scientific breakthrough are the fluctuations of the cosmic microwave background radiation, first carried out by the COBE satelite and later refine by WMAP. In the case of the cosmic microwave background, the fluctuations are on the level of $10^{-4}$ with respect to the thermal distribution. In addition a large dipole correlation, due to the motion of the earth through the heatbath of the microwave background, as been seen and needed to be subtracted before the more intersting correlations due to the big bang could be revealed.

In the cosmic microwave background one observes spatial correlations. In contrast, in heavy ion collisions we are restricted to correlations in momenta and the connection to spatial correlations is in general non-trivial. Nonetheless, in case of heavy ion collisions we have quite a similar situation. To first order we observe thermal spectra and radial flow. Next, we see a large quadrupole correlation due to elliptic flow. The interesting question then remains, if we will be able to identify smaller correlations due to a possible phase transition and the QCD critical point after subtracting the two dominant backgrounds, thermal emission and elliptic flow.

For instance a second order phase transition, such as the QCD critical point, will give rise to correlations and fluctuations at all length scales, and thus should be visible in a correlation analysis (see the contribution of M. Stephanov to these proceedings [1]). Of course a system produced in heavy ion collisions is a very dynamical one, and there will not be time to develop the correlations at all length scales. But there should be sufficient time to build up correlation over several Fermi [2]. 


\section{Thermal fluctuations}

Before we discuss the subtleties of heavy ion collisions, it is best to first introduce the concepts of fluctuations and correlations for a thermal/statistical system, where things are well defined. Besides, all Lattice QCD (LQCD) results for fluctuations and correlations are obtained for thermal systems.

A statistical/thermal system (for a grand-canonical ensemble) is characterized by its partition function

$$
Z=\operatorname{Tr}\left[\exp \left(-\frac{H-\sum_{i} \mu_{i} Q_{i}}{T}\right)\right]
$$

where $H$ is Hamiltonian of the system, and $Q_{i}$ and $\mu_{i}$ denote the conserved charges and the corresponding chemical potentials, respectively. In case of three flavor QCD these are strangeness, baryon-number, and electric charge. The mean and the (co)variances are then expressed in terms of derivatives of the partition function with respect to the chemical potentials

$$
\begin{aligned}
& \left\langle Q_{i}\right\rangle=T \frac{\partial}{\partial \mu_{i}} \log (Z) \\
& \left\langle\left(\delta Q_{i} \delta Q_{j}\right\rangle=T^{2} \frac{\partial^{2}}{\partial \mu_{i} \partial \mu_{j}} \log (Z) \equiv V T \chi_{i, j}\right.
\end{aligned}
$$

with $\delta Q=Q_{i}-\left\langle Q_{i}\right\rangle$. Here we have introduced the susceptibilities

$$
\chi_{i, j}=\frac{T}{V} \frac{\partial^{2}}{\partial \mu_{i} \partial \mu_{j}} \log (Z)
$$

which are generally quoted as a measure of the (co)-variances. The diagonal susceptibilities, $\chi_{i, i}$, are a measure for the fluctuations of the system, whereas the offdiagonal susceptibilities, $\chi_{i, j} i \neq j$, characterize the correlations between the conserved charges $Q_{i}$ and $Q_{j}$. The susceptibilities are integrals over the correlation functions of the appropriate charge (strangeness, baryon number of electric charge) densities

$$
\chi_{i, j}=\frac{T}{V} \int d^{3} x d^{3} y\left\langle\delta \rho_{i}(x) \delta \rho_{j}(y)\right\rangle=T \bar{\rho}_{i} \delta_{i, j}+T \int d^{3} r C_{i, j}(r) .
$$

where $\bar{\rho}_{i}$ is the mean density of the charge $Q_{i}$ and $\delta \rho(x)=\rho(x)-\bar{\rho}$ denotes a density fluctuation at location $x$. The correlation functions

$$
C_{i, j}(\vec{r})=\left\langle\delta \rho_{i}(\vec{r}) \delta \rho_{j}(0)\right\rangle-\bar{\rho}_{i} \delta_{i, j} \delta(\vec{r}) \sim \frac{\exp \left[-r / \xi_{i, j}\right]}{r}
$$

are usually characterized by the correlation lengths $\xi_{i, j}$, which in turn provide a measure for the susceptibilities. While we will concentrate here on susceptibilities involving conserved charges, we note that one can define susceptibilities involving any well defined operator. One prominent example is the chiral susceptibility $\chi_{c h}=T / V \partial^{2} / \partial m_{q}^{2} \log (Z)$ which characterizes the chiral phase transition in QCD.

For example, the correlation function of a classical ideal gas vanishes, $C_{\text {ideal gas }}=$ 0 , and the susceptibilities are given by the first, local term $\sim \bar{\rho}_{i} \delta_{i, j}$, implying that all co-variances vanish. As a consequence the fluctuations are proportional to the number of particles in the system, and thus grow linearly with the system size.

The more relevant case concerning the QCD critical point corresponds to a second order phase transition. In this case the correlation length diverges at the critical temperature

$$
\xi \sim\left|T-T_{c}\right|^{-\nu}
$$


where $\nu>0$ is one of the critical exponents characterizing a second order phase transition in a given universality class [3]. In this case the volume dependence of the susceptibilities is governed by the integral of the correlation function

$$
\chi_{i, j} \sim \xi^{2} \sim V^{2 / 3}
$$

so that the fluctuations grow likeł

$$
\left\langle\left(\delta Q_{i}\right)\right\rangle \sim V^{5 / 3} \text {, second order. }
$$

In case of a first order transition we have co-existence of phases with different densities, and the correlation function is a constant, $C(r)=$ const $\neq 0$. Consequently the fluctuations scale like

$$
\left\langle\left(\delta Q_{i}\right)\right\rangle \sim V^{2}, \text { first order. }
$$

Most other systems, including systems with a cross over, such as QCD at vanishing chemical potential [4], will exhibit a finite correlation length. Consequently, the susceptibility is independent of the volume, and the fluctuations scale linearly with the volume, just as in the case of an ideal gas

$$
\left\langle\left(\delta Q_{i}\right)\right\rangle \sim V, \text { No phase - transition. }
$$

In principle, one could utilize the above volume scaling of the fluctuations in heavy ion experiments by studying the system size dependence of e.g. baryon-number fluctuations. However, in case of the second order phase-transition the phenomenon of critical slowing down limits the actual size of correlation length due to the finite life-time of the system created in these collisions. As already mentioned, a maximum correlation length of $\xi \sim 2 \mathrm{fm}$ has been estimated in ref. [2] which is much smaller than the typical size of a system created in these reactions. Consequently, such a system would just behave like any other with a constant correlation-length and, therefore, would not exhibit the system size dependence discussed above.

The above susceptibilities of conserved charges can be and have been calculated in Lattice QCD $[5,6,7]$ where even higher order derivatives of the free energy with respect to the chemical potential have been calculated. These correspond to higher moments of the appropriate charge operator. In addition, some of the off-diagonal susceptibilities have been determined on the lattice [5], and it has been shown [8] that they, together with higher moments for the baryon-number [9], provide important information about the relevant degrees of freedom of a QGP.

Since the susceptibilities measure the fluctuations of the charges, they are in principle accessible to experiment by studying event-by-event fluctuations of these relevant net charge [10]. An overview of the experimental status of fluctuation measurements is given in the contribution by G. Westfall in these proceedings [11].

\section{Fluctuations of conserved charges}

Quite a number of experimental studies involve the fluctuations of conserved quantities. Therefore, we will briefly review the general ideas behind these measurements and also point out some of the caveats when going to lower energies in the quest for the QCD critical point and first order co-existence region.

As first pointed out in refs. $[12,13]$ the measurement of electric charge fluctuations is sensitive to the fractional charge of the quarks and thus to the existence of the

$\ddagger$ The correct scaling of the susceptibility with the volume actually involves the critical exponents. Our example here is correct for so called mean field exponent. For details see [3]. 
QGP. Naturally, fluctuations of a conserved charge are only a sensible probe if a small subsystem is considered since the total charge of the system is conserved and, therefore, does not fluctuate. On the other hand, the conservation of the charge ensures that the total charge within a given rapidity interval can only change by transport of charges in and out of this interval. This actually leads to a rather large equilibration time for the charge fluctuations in the hadronic phase and allows, at least in principle, to detect the initial charge fluctuations from the QGP [10,14, 15]. This argument is governed by four rapidity ranges/scales, which need to be well separated:

- The range $\Delta Y_{\text {Total }}$ for the total charge multiplicity distribution.

- The interval $\Delta Y_{\text {accept }}$ for the accepted charged particles.

- The charge correlation length $\Delta Y_{\text {corr }}$ characteristic to the physics of interest.

- The typical rapidity shift $\Delta Y_{k i c k}$ charges receive during and after hadronization.

Given these scales, charge fluctuations will be able to tell us about the properties of the early stage of the system, the QGP, if the following criteria are met:

$$
\begin{aligned}
& \Delta Y_{\text {accept }} \gg \Delta Y_{\text {corr }} \\
& \Delta Y_{\text {Total }} \gg \Delta Y_{\text {accept }} \gg \Delta Y_{\text {kick }}
\end{aligned}
$$

The first criterion, Eq. 12, is necessary to be sensitive to the relevant physics, whereas the second one, Eq. 13, ensures that total charge conservation does not suppress the signal and that the signal survives hadronization and the hadronic phase§.

Consequently, for sufficiently high bombarding energies and a sufficiently large acceptance one should be able to see "back" into the QGP, provided that hadronization leads to a limited distribution of charge kicks, such that one can assign a typical scale $\Delta Y_{\text {kick }}$. The charge kick due to re-scattering in the hadronic phase can be estimated using transport models and one finds [15] $\Delta Y_{k i c k} \simeq 2$, close to a naive estimate from $\rho$ decays, which gives $\Delta Y_{k i c k} \simeq 1.5$. An estimate of the charge transport during hadronization is very difficult on the other hand. If, for example, the charge kicks are distributed according to a power law, the above considerations are invalid, as the charge transport involves the entire system. This might very well be the reason for the results of $[17,18]$ where in a quark molecular dynamics model, the charge fluctuations quickly adjust to the hadron gas value during hadronization. We note, that in this model string breaking during hadronization was not taken into account, allowing for charge transport over large rapidity intervals due to the linearly increasing potential among the quarks.

Finally we should note, that present measurements at RHIC and SPS have not found the small net-charge fluctuations predicted for a QGP $[12,13]$ and are rather consistent with a resonance gas (for details see [11]).

Concerning the QCD critical point, where the measurement of baryon-number fluctuations has been proposed as one possible signature [19], the above criteria are seriously violated, calling into question the proper interpretation of such a measurement. The minimal charge kick is $\Delta Y_{\text {kick }} \geq 1.5$ from the decay of hadronic resonance such as the rho-meson and the delta-resonance. This is comparable with the width $\Delta Y_{\text {Total }} \simeq 2$ of the multiplicity distribution at top SPS energies. This complicates the interpretation of net charge fluctuations at these energies [20]. Going

$\S$ The treatment and correction for the total charge conservation is non-trivial and needs to be done carefully [10] since the variance in a grand-canonical and canonical ensemble differ even for large systems [16]. 
to even lower energies in order to search for the critical point and first order phase coexistence, implies $\Delta Y_{k i c k} \simeq \Delta Y_{\text {Total }}$ and a interpretation of the measured fluctuations becomes rather difficult.

\section{Observables}

In this section we want to concentrate on observables relevant for QCD critical point and phase co-existence region. For a status of present experimental results we refer to the contribution by G. Westfall [11].

Since the baryon density is a an order parameter for the phase transition at finite density, baryon number fluctuations are the natural observable to consider. In the previous section, we have already discussed the serious limitations baryon number conservation imposes on this observable.

However, global baryon number conservation does not prevent the system from having large, local (in space) fluctuations of the baryon density. Therefore, one needs to devise observables which are sensitive to these local density fluctuations. Since the final state will be a volume average of these density fluctuations, the observable has to have a nonlinear dependence on the baryon density. Otherwise, baryon number conservation becomes an issue again. One example would be dilepton production, where the broadening of the spectral function depends on higher powers of the baryon density. Thus one would expect an increased broadening of the electromagnetic spectral function close to the critical point.

Another observable often discussed in the literature is to look for soft modes, such as dilepton production at very low invariant masses. However, it is not clear at all if the soft modes close to the critical point will be visible as a signal in the low mass dilepton spectrum. The modes responsible for the large density fluctuations are of space like origin and, although they carry the quantum numbers of the omega meson, they don't contribute to the time like dilepton-spectrum. Indeed, an analysis of the fluctuations close to the critical point carried out in the Nambu model with finite quark masses $[21,22]$ showed that the sigma-meson remained gaped at the critical point, contrary to the chiral transition in the limit of vanishing quark masses. At the same time, the authors found, that the relevant fluctuations driving the phase transition are those of the baryon density, as in a regular liquid gas transition. This is also consistent with the arguments of [23] who identified the QCD critical point to be in the same universality class as a liquid gas transition. While the fluctuations of the chiral order parameter also diverge in the aforementioned model study, this was simply due to the coupling between the chiral order parameter and the baryon density.

In this context, it maybe interesting to further explore the co-variances between the baryon density fluctuations and other quantities which couple to the baryon density. These co-variances are expected to become large and could possibly be developed into practical observables, which will not be affected by baryon number conservation.

Let us close this section on observables by stressing that it might be more beneficial to first look for and identify the first order co-existence region. Contrary to the critical point the first order transition corresponds to an entire region in the $T-\rho$ phase diagram. Thus it is more likely for the system to cross this region rather than the critical point. It is also more likely for the system to spend sufficient time in this region in order to develop measurable effects. One example is the development of spinodal instabilities, which are a generic phenomenon of dynamical first order 
transitions [24]. In case of the QCD first order transition, spinodal instabilities could lead to kinematic correlations of particles [25] and possibly to enhanced fluctuations of strangeness [26]. The consequences of these spinodal instabilities need to be explored much further and it may very well be that they will turn out equally important for the QCD matter transition as for the regular liquid gas transition [24].

\section{Conclusions}

Fluctuations are an essential property of any physical system and they are important signatures and characteristics of systems near a phase transition. While first measurements at SPS and RHIC have been mostly consistent with either statistical fluctuations or correlations due to resonance decay, we note that the fluctuations of the kaon to pion ratio observed by NA49 shows a very interesting rise as the beam energy is lowered to $\simeq 20 \mathrm{AGeV}$ [27]. Interestingly for the same energy region, $20 \mathrm{AGeV} \leq E_{\text {Beam }} \leq 40 \mathrm{AGeV}$ the inclusive kaon to pion ratio increases as well. And so does the anti-lamda to anti-proton ratio. While the latter two may be explained either by fine tuning the statistical model [28] or by acceptance cuts [29], respectively, the large fluctuations of the kaon-to-pion ratio so far cannot be understood in conventional terms, such as the statistical model [30]. Incidentally, it is this energy around $E_{\text {Beam }} \simeq 30 \mathrm{AGeV}$ where the highest baryon density is reached. Certainly these large fluctuations in the kaon to pion ratio, if confirmed, are very intriguing and need to be further explored. At present RHIC is planning a low energy run to exactly address these issues and the CBM experiment at the new FAIR facility will be able to study the physics close to the QCD phase boundary with unprecedented precision.

On the theoretical side, Lattice QCD make considerable progress. New methods are being developed to explore the region of finite baryon chemical potential (see contributions by P. deForcrand, S. Gupta, C. Schmidt to these proceedings). Also simulations with almost realistic quark masses are now possible, and the first results on susceptibilities show, for the first time, deviations from the hadron gas below the critical temperature [31]. Although some progress is being made, there is still a desperate need for robust observables which are sensitive to the critical point and the co-existence region. Also dynamical models need to be developed, which are able to describe the evolution of the system through the critical and/or co-existence region.

While the field is preparing for the next leap in energy by setting up at the LHC, it will be equally if not more interesting to carefully revisit the high density regime. In particular if one wants to identify possible structures of the QCD phase diagram, this is the more promissing direction, since Lattice QCD has by now established that at vanishing baryon density QCD exhibits a cross over transition [4].

\section{Acknowledgments}

This work is supported by the Director, Office of Energy Research, Office of High Energy and Nuclear Physics, Divisions of Nuclear Physics, of the U.S. Department of Energy under Contract No. DE-AC02-05CH11231.

\section{References}

[1] M. Stephanov. These proceedings. 
[2] Boris Berdnikov and Krishna Rajagopal. Slowing out of equilibrium near the qcd critical point. Phys. Rev., D61:105017, 2000.

[3] L Landau and L.M. Lifshitz. Statistical Physics. Pergamon Press, New York, 1980.

[4] Y. Aoki, G. Endrodi, Z. Fodor, S. D. Katz, and K. K. Szabo. The order of the quantum chromodynamics transition predicted by the standard model of particle physics. Nature, 443:675-678, 2006

[5] R. V. Gavai and Sourendu Gupta. Fluctuations, strangeness and quasi-quarks in heavy-ion collisions from lattice qcd. Phys. Rev., D73:014004, 2006.

[6] Rajiv V. Gavai and Sourendu Gupta. The continuum limit of quark number susceptibilities. Phys. Rev., D65:094515, 2002.

[7] C. R. Allton et al. Thermodynamics of two flavor qcd to sixth order in quark chemical potential. Phys. Rev., D71:054508, 2005.

[8] V. Koch, A. Majumder, and J. Randrup. Baryon-strangeness correlations: A diagnostic of strongly interacting matter. Phys. Rev. Lett., 95:182301, 2005.

[9] S. Ejiri, F. Karsch, and K. Redlich. Hadronic fluctuations at the QCD phase transition. Phys. Lett., B633:275-282, 2006.

[10] S Jeon and V Koch. Event-by-event fluctuations. In R Hwa and X.N. Wang, editors, Quark Gluon Plasma 3, pages 430-490. World Scientific, Singapore, 2004.

[11] G. Westfall. These proceedings.

[12] Masayuki Asakawa, Ulrich W. Heinz, and Berndt Muller. Fluctuation probes of quark deconfinement. Phys. Rev. Lett., 85:2072-2075, 2000.

[13] S. Jeon and V. Koch. Charged particle ratio fluctuation as a signal for qgp. Phys. Rev. Lett., 85:2076-2079, 2000.

[14] M. Bleicher, S. Jeon, and V. Koch. Event-by-event fluctuations of the charged particle ratio from non-equilibrium transport theory. Phys. Rev., C62:061902, 2000.

[15] Edward V. Shuryak and Misha A. Stephanov. When can long range charge fluctuations serve as a qgp signal? Phys. Rev., C63:064903, 2001.

[16] V. V. Begun and Mark I. Gorenstein. Particle number fluctuations in relativistic Bose and Fermi gases. Phys. Rev., C73:054904, 2006.

[17] Stephane Haussler, Stefan Scherer, and Marcus Bleicher. The effect of dynamical parton recombination on event-by- event observables. Phys. Lett., B660:197-201, 2008.

[18] Stephane Haussler, Marcus Bleicher, and Horst Stocker. Susceptibilities and fluctuations in a Quark-Hadron System with Dynamical Recombination. 2008.

[19] Y. Hatta and M. A. Stephanov. Proton number fluctuation as a signal of the QCD critical end-point. Phys. Rev. Lett., 91:102003, 2003.

[20] Jacek Zaranek. Measures of charge fluctuations in nuclear collisions. Phys. Rev., C66:024905, 2002.

[21] H. Fujii and M. Ohtani. Mode softening near the critical point within effective approaches to QCD. 2004.

[22] H. Fujii and M. Ohtani. Soft modes at the critical end point in the chiral effective models. Prog. Theor. Phys. Suppl., 153:157-164, 2004.

[23] D. T. Son and M. A. Stephanov. Dynamic universality class of the QCD critical point. Phys. Rev., D70:056001, 2004.

[24] Philipe Chomaz, Maria Colonna, and Jorgen Randrup. Nuclear spinodal fragmentation. Phys. Rept., 389:263-440, 2004.

[25] J. Randrup. Probes of phase decomposition in high-energy nuclear collisions. Acta Phys. Hung., A22:69-82, 2005.

[26] Volker Koch, Abhijit Majumder, and Jorgen Randrup. Signals of spinodal hadronization: Strangeness trapping. Phys. Rev., C72:064903, 2005.

[27] C. Roland. Event-by-event fluctuations of particle ratios in central $\mathrm{Pb}+\mathrm{Pb}$ collisions at 20-AGeV - 158-A-GeV. J. Phys., G31:S1075-S1078, 2005.

[28] F. Becattini, M. Gazdzicki, A. Keranen, J. Manninen, and R. Stock. Study of chemical equilibrium in nucleus nucleus collisions at AGS and SPS energies. Phys. Rev., C69:024905, 2004.

[29] Fuqiang Wang. Strangeness in dense nuclear matter: A review of AGS results. J. Phys., G27:283-300, 2001.

[30] Giorgio Torrieri. A statistical model analysis of $K / \pi$ fluctuations in heavy ion collisions. Int. J. Mod. Phys., E16:1783-1789, 2007.

[31] C. Schmidt. These proceedings. 